PROCEEDINGS OF THE

AMERICAN MATHEMATICAL SOCIETY

Volume 133, Number 1, Pages 127-131

S 0002-9939(04)07476-3

Article electronically published on August 20, 2004

\title{
A LIPSCHITZ ESTIMATE FOR BEREZIN'S OPERATOR CALCULUS
}

\author{
L. A. COBURN
}

(Communicated by Joseph A. Ball)

\begin{abstract}
F. A. Berezin introduced a general "symbol calculus" for linear operators on reproducing kernel Hilbert spaces. For the particular Hilbert space of Gaussian square-integrable entire functions on complex $n$-space, $\mathbf{C}^{n}$, we obtain Lipschitz estimates for the Berezin symbols of arbitrary bounded operators. Additional properties of the Berezin symbol and extensions to more general reproducing kernel Hilbert spaces are discussed.
\end{abstract}

\section{INTRODUCTION}

The Segal-Bargmann Hilbert space $H^{2}\left(\mathbf{C}^{n}, d \mu\right)$ of Gaussian square-integrable entire functions on complex $n$-space [Ba] has the Bergman "reproducing kernel" property that

$$
f(a)=\int_{\mathbf{C}^{n}} K(a, z) f(z) d \mu(z)=\langle f(\cdot), K(\cdot, a)\rangle
$$

for all $f$ in $H^{2}\left(\mathbf{C}^{n}, d \mu\right)$ and $a$ in $\mathbf{C}^{n}$ where $a \cdot z=a_{1} \bar{z}_{1}+\cdots+a_{n} \bar{z}_{n},|a|^{2}=a \cdot a$, $K(a, z)=e^{a \cdot z / 2}$ is the Bergman kernel function and

$$
d \mu(z)=(2 \pi)^{-n} \exp \left\{-|z|^{2} / 2\right\} d v(z)
$$

where $d v$ is the Lebesgue volume measure. It is easy to check that $k_{a}(z)=$ $K(z, a)\{K(a, a)\}^{-\frac{1}{2}}$ is a unit vector in the Hilbert space structure that $H^{2}\left(\mathbf{C}^{n}, d \mu\right)$ inherits as a subspace of $L^{2}\left(\mathbf{C}^{n}, d \mu\right)$.

Although we will deal primarily with the space $H^{2}\left(\mathbf{C}^{n}, d \mu\right)$, we will also consider the analogous Bergman spaces $A^{2}(\Omega)$ for $\Omega$ a bounded domain in $\mathbf{C}^{n}$ and the Gaussian $d \mu$ replaced by $d v$. Their kernel functions will also be denoted by $K(a, z)$ as in $[\mathrm{K}$, pp. 39-54]. Note that $K(a, z)$ is always analytic in $a$ and conjugate-analytic in $z$ with

$$
\overline{K(a, z)}=K(z, a)
$$

and that $K(a, a)$ is positive. The "bounded symmetric domains" $\Omega$ give interesting special cases that are "closer" to the model $H^{2}\left(\mathbf{C}^{n}, d \mu\right)[\mathrm{H}]$.

For bounded linear operators $X$ on $H^{2}\left(\mathbf{C}^{n}, d \mu\right)$, or, more generally, linear operators $X$ such that $X$ and $X^{*}$ have domains that include all the $\left\{k_{a}: a \in\right.$ $\mathbf{C}^{n}$, Berezin $\mathrm{Be}_{1}, \mathrm{Be}_{2}$ considered the mapping $\operatorname{Ber}: X \rightarrow \tilde{X}$, where $\tilde{X}(a)=$ $\left\langle X k_{a}, k_{a}\right\rangle$. The function $\tilde{X}(\cdot)$ is real-analytic on $\mathbf{C}^{n}$ and is uniquely determined by

Received by the editors July 8, 2003 and, in revised form, August 15, 2003 and September 5, 2003.

2000 Mathematics Subject Classification. Primary 47B32; Secondary 32A36. 
$X\left[\overline{\mathrm{Be}_{1}}\right] ;[\mathrm{F}$, pp. 43, 139]. It is clear that $|\tilde{X}(\cdot)| \leq\|X\|$, where $\|\cdot\|$ is the usual operator norm. Berezin's map has the above properties for any of the spaces $A^{2}(\Omega)$. Because Ber is linear and one-to-one, it "encodes" operator-theoretic information into function-theory in a striking but somewhat impenetrable way. In fact, since $k_{a} \rightarrow 0$ weakly as $|a| \rightarrow \infty$ for $H^{2}\left(\mathbf{C}^{n}, d \mu\right)$, it is also clear that Ber maps compact operators on these Hilbert spaces into functions that vanish at infinity (there is an analogous result for $A^{2}(\Omega)$ with "nice" $\Omega$ ). Because of these properties, the mapping $B e r$ has found useful applications in dealing with operators "of function-theoretic significance" such as Toeplitz and Hankel operators $\mathrm{AZ}, \mathrm{BBCZ}, \mathrm{BC}_{1}, \mathrm{BC}_{2}, \mathrm{G}, \mathrm{E}$.

In the next section, I will prove the promised result that $\tilde{X}$ is Lipschitz for arbitrary bounded operators $X$ on $H^{2}\left(\mathbf{C}^{n}, d \mu\right)$. I will also consider extensions of this result to more general settings. In the third section of the paper, I will discuss some other properties of the range of Ber.

\section{The Lipschitz estimate for Ber}

Let $P_{a}(f)=\left\langle f, k_{a}\right\rangle k_{a}$ be the rank one projection onto the span of $k_{a}$. We will need a few technical results about trace-class operators, which we take from GK]. For $X$ any bounded linear operator on $H^{2}\left(\mathbf{C}^{n}, d \mu\right), X P_{a}$ is also rank one and, therefore, trace-class. Moreover, since we can augment $k_{a}$ to an orthonormal basis for $H^{2}\left(\mathbf{C}^{n}, d \mu\right)$, it is easy to see that

$$
\operatorname{trace}\left(X P_{a}\right)=\left\langle X P_{a} k_{a}, k_{a}\right\rangle=\tilde{X}(a) .
$$

Additivity of the trace now shows that

$$
\tilde{X}(a)-\tilde{X}(b)=\operatorname{trace}\left[X\left(P_{a}-P_{b}\right)\right] .
$$

We need to calculate $\left\|P_{a}-P_{b}\right\|_{\text {trace }}$, where GK, pp. 47, 48, 92]

$$
\|A\|_{\text {trace }}=\operatorname{trace}\left(\sqrt{A^{*} A}\right)
$$

and $\sqrt{A^{*} A}$ is the unique positive square-root of the positive operator $A^{*} A$. This calculation holds for any reproducing kernel space.

Theorem 1. For $a, b$ in $\mathbf{C}^{n}$,

$$
\left\|P_{a}-P_{b}\right\|_{\text {trace }}=2\left\{1-\left|\left\langle k_{a}, k_{b}\right\rangle\right|^{2}\right\}^{\frac{1}{2}} .
$$

Proof. We write $(g \otimes h)(f)=\langle f, h\rangle g$ for a typical rank-one operator. Now

$$
k_{b}=\left\langle k_{b}, k_{a}\right\rangle k_{a}+h_{a, b}
$$

with $\left\langle h_{a, b}, k_{a}\right\rangle=0$ and

$$
\left\|h_{a, b}\right\|^{2}=1-\left|\left\langle k_{a}, k_{b}\right\rangle\right|^{2}
$$

Direct calculation shows that

$$
\begin{aligned}
P_{a}-P_{b}=\left\|h_{a, b}\right\|^{2} k_{a} & \otimes k_{a}-\left\langle k_{a}, k_{b}\right\rangle h_{a, b} \otimes k_{a} \\
& -\left\langle k_{b}, k_{a}\right\rangle k_{a} \otimes h_{a, b}-h_{a, b} \otimes h_{a, b} .
\end{aligned}
$$

Now $P_{a}-P_{b}$ is self-adjoint and another direct calculation shows that

$$
\left(P_{a}-P_{b}\right)^{2}=\left\|h_{a, b}\right\|^{2} k_{a} \otimes k_{a}+h_{a, b} \otimes h_{a, b} .
$$

Thus, $P_{a}-P_{b}=0$ if and only if $h_{a, b}=0$. For $h_{a, b} \neq 0,\left(P_{a}-P_{b}\right)^{2}$ is diagonal in any orthonormal basis including $k_{a}$ and $h_{a, b} /\left\|h_{a, b}\right\|$ and has two non-zero eigenvalues, 
both equal to $\left\|h_{a, b}\right\|^{2}$. The positive square root of $\left(P_{a}-P_{b}\right)^{2}$ is then diagonal in the same basis and it follows that

$$
\left\|P_{a}-P_{b}\right\|_{\text {trace }}=2\left\|h_{a, b}\right\|=2\left\{1-\left|\left\langle k_{a}, k_{b}\right\rangle\right|^{2}\right\}^{\frac{1}{2}} .
$$

Remark. Since $P_{a}-P_{b}=0$ implies that $\left|\left\langle k_{a}, k_{b}\right\rangle\right|=1$, it is an easy consequence of the Cauchy-Schwarz Lemma that $K(\cdot, a)=\lambda K(\cdot, b)$ for some complex number $\lambda$ and an easy argument shows, for $A^{2}(\Omega)$ with $\Omega$ bounded or for $H^{2}\left(\mathbf{C}^{n}, d \mu\right)$, that $a=b$. Subadditivity of $\|\cdot\|_{\text {trace }}$ now implies that $d(a, b)=\left\|P_{a}-P_{b}\right\|_{\text {trace }}$ is a topological metric (distance function) on bounded $\Omega$ or $\mathbf{C}^{n}$. For bounded $\Omega$, it is easy to check, using the "transformation laws for reproducing kernels" [K, p. 44], that $\left|\left\langle k_{\varphi(a)}, k_{\varphi(b)}\right\rangle\right|^{2}=\left|\left\langle k_{a}, k_{b}\right\rangle\right|^{2}$ for $\varphi$ any biholomorphic automorphism of $\Omega$ and it follows that $\left\|P_{\varphi(a)}-P_{\varphi(b)}\right\|_{\text {trace }}=\left\|P_{a}-P_{b}\right\|_{\text {trace }}$.

We can now establish the desired Lipschitz estimate.

Theorem 2. For $X$ any bounded operator on $H^{2}\left(\mathbf{C}^{n}, d \mu\right), \tilde{X}$ is Lipschitz with

$$
|\tilde{X}(a)-\tilde{X}(b)| \leq \sqrt{2}\|X\||a-b|
$$

Proof. As noted earlier,

$$
\tilde{X}(a)-\tilde{X}(b)=\operatorname{trace}\left[X\left(P_{a}-P_{b}\right)\right] .
$$

For $X$ bounded and $T$ in trace-class, it is known [GK, pp. 27, 104] that $X T$ is in trace-class with

$$
|\operatorname{trace}(X T)| \leq\|X\|\|T\|_{\text {trace }} .
$$

It follows from Theorem 1 that

$$
|\tilde{X}(a)-\tilde{X}(b)| \leq 2\|X\|\left\{1-\left|\left\langle k_{a}, k_{b}\right\rangle\right|^{2}\right\}^{\frac{1}{2}} .
$$

By direct calculation, using $K(z, a)=e^{z \cdot a / 2}$, we see that

$$
1-\left|\left\langle k_{a}, k_{b}\right\rangle\right|^{2}=1-e^{-|a-b|^{2} / 2} \leq \frac{|a-b|^{2}}{2},
$$

and the proof is finished.

The proof of Theorem 1 holds in general reproducing kernel spaces. It is known that the Bergman kernel function induces a Riemannian metric on $\Omega$ or $\mathbf{C}^{n}$ and this, in turn, gives rise to a distance function $\beta(\cdot, \cdot)$. For $H^{2}\left(\mathbf{C}^{n}, d \mu\right)$, we can check that $\beta(a, b)=|a-b|$. Using results of [MPS], we can obtain the exact analogue of Theorem 2 for $A^{2}(\Omega)$ with $\Omega$ any bounded domain in $\mathbf{C}^{n}$.

Theorem 3. For $X$ any bounded operator on $A^{2}(\Omega)$ with $\Omega$ any bounded domain in $\mathbf{C}^{n}, \tilde{X}$ is " $\beta$-Lipschitz" with

$$
|\tilde{X}(a)-\tilde{X}(b)| \leq \sqrt{2}\|X\| \beta(a, b)
$$

Proof. By Theorem 1 (which holds without change in the proof) and [MPS, p. 73], we have

$$
\begin{aligned}
\left\|P_{a}-P_{b}\right\|_{\text {trace }} & =2\left\{1-\left|\left\langle k_{a}, k_{b}\right\rangle\right|^{2}\right\}^{\frac{1}{2}} \\
& \leq 2\left\{1+\left|\left\langle k_{a}, k_{b}\right\rangle\right|\right\}^{\frac{1}{2}}\left\{1-\left|\left\langle k_{a}, k_{b}\right\rangle\right|\right\}^{\frac{1}{2}} \\
& \leq 2 \sqrt{2}\left\{1-\left|\left\langle k_{a}, k_{b}\right\rangle\right|\right\}^{\frac{1}{2}} \\
& \leq \sqrt{2} \beta(a, b) .
\end{aligned}
$$


The equation

$$
\tilde{X}(a)-\tilde{X}(b)=\operatorname{trace}\left[X\left(P_{a}-P_{b}\right)\right]
$$

leads to the desired result, as in the proof of Theorem 2 .

Corollary. For $\psi$ any bounded analytic function on the bounded domain $\Omega$ in $\mathbf{C}^{n}$ and for $a, b$ any two points in $\Omega$, we have

$$
\begin{aligned}
|\psi(a)-\psi(b)| & \leq 2\|\psi\|_{\infty}\left\{1-\left|\left\langle k_{a}, k_{b}\right\rangle\right|^{2}\right\}^{\frac{1}{2}} \\
& \leq \sqrt{2}\|\psi\|_{\infty} \beta(a, b) .
\end{aligned}
$$

Proof. Take $X$ to be "multiplication by $\psi$ " on $A^{2}(\Omega)$.

Remark. I thank Miroslav Engliš for calling [MPS] to my attention.

\section{The Range of Ber}

We now restrict our attention to the Hilbert space $H^{2}\left(\mathbf{C}^{n}, d \mu\right)$. The linear map $B e r: X \rightarrow \tilde{X}$ is defined and one-to-one on all linear operators $X$ such that $X$ and $X^{*}$ have domains that include all the $\left\{k_{a}: a \in \mathbf{C}^{n}\right\}$. We denote the algebra of all bounded (and everywhere defined) linear operators on $H^{2}\left(\mathbf{C}^{n}, d \mu\right)$ by $O p$. The range $\operatorname{Ber}(O p)$ is not well-understood. By our earlier discussion, it is a linear space of bounded real-analytic Lipschitz functions on $\mathbf{C}^{n}$. On the positive side, we have

Theorem 4. $\operatorname{Ber}(O p)$ is translation-invariant.

Proof. For all $c$ in $\mathbf{C}^{n}$, we consider the unitary operators $\left(W_{c} f\right)(z)=k_{c}(z) f(z-c)$. It is easy to check that

$$
W_{c} k_{a}=e^{i \operatorname{Im}(a \cdot c) / 2} k_{a+c} .
$$

It follows that, for any $X$ in $O p$,

$$
\left\langle W_{c}^{*} X W_{c} k_{a}, k_{a}\right\rangle=\left\langle X k_{a+c}, k_{a+c}\right\rangle,
$$

and so the desired result follows.

Remark. Jingbo Xia has pointed out to me that $(*)$ in the proof of Theorem 4 can be used, along with a direct calculation, to provide a strengthened version of Theorem 2: for any $X$ in $O p\left\{H^{2}\left(\mathbf{C}^{n}, d \mu\right)\right\}, \tilde{X}$ and its partial derivatives of all orders are bounded. This version of the Lipschitz estimate depends upon the special properties of the $\left\{W_{c}\right\}$ which have no general analogue, even for bounded symmetric $\Omega$.

We close with a result which shows that very nice, bounded, smooth Lipschitz functions may fail to be in $\operatorname{Ber}(O p)$.

Theorem 5. There is no $X$ in $O p$ with $\tilde{X}(a)=e^{-2|a|^{2}}$.

Proof. For $t$ real, consider the linear operators on $H^{2}\left(\mathbf{C}^{n}, d \mu\right)$ given by

$$
\left(R_{t} f\right)(z)=f(t z) .
$$

The operator $R_{t}$ is bounded for $|t| \leq 1$ and unbounded otherwise. By direct computation,

and

$$
R_{t} k_{a}=k_{a t} e^{-|a|^{2}\left(1-t^{2}\right) / 4}
$$

$$
\tilde{R}_{t}(a)=e^{|a|^{2}(t-1) / 2} .
$$

Since $\left\langle R_{t} k_{a}, k_{b}\right\rangle=\left\langle k_{a}, R_{t} k_{b}\right\rangle$ for all $a, b$ in $\mathbf{C}^{n}$, it follows that $R_{t}^{*} k_{a}=R_{t} k_{a}$. 
Now suppose there is a bounded operator $X$ with

$$
\tilde{X}(a)=e^{-2|a|^{2}} .
$$

Then $A=X-R_{-3}$ has the property that $A k_{a}, A^{*} k_{a}$ are all in $H^{2}\left(\mathbf{C}^{n}, d \mu\right)$ and $\tilde{A}(a) \equiv 0$. It follows that $A k_{a}=X k_{a}-R_{-3} k_{a}=0$ and so

$$
X k_{a}=k_{-3 a} e^{2|a|^{2}} .
$$

This contradicts the boundedness of $X$.

\section{REFERENCES}

[AZ] S. Axler and D. Zheng, Compact operators via the Berezin transform, Indiana Univ. Math. J., 47 (1998), 387-400. MR1647896 (99i:47045)

[Ba] V. Bargmann, On a Hilbert space of analytic functions and an associated integral transform, Communications on Pure and Applied Mathematics, 14 (1961), 187-214. MR0157250 (28:486)

$\left[\mathrm{Be}_{1}\right] \quad$ F. A. Berezin, Covariant and contravariant symbols of operators, Math. USSR Izv., 6 (1972), 1117-1151. MF0350504 (50:2996)

$\left[\mathrm{Be}_{2}\right] \quad$ F. A. Berezin, Quantization, Math. USSR Izv., 8 (1974), 1109-1163. MR0395610 $(52: 16404)$

[BBCZ] D. Békollé, C. A. Berger, L. A. Coburn, and K. H. Zhu, BMO in the Bergman metric on bounded symmetric domains, J. Funct. Anal., 93 (1990), 310-350. MR1073289 (91j:32034)

$\left[\mathrm{BC}_{1}\right]$ C. A. Berger and L. A. Coburn, Toeplitz operators on the Segal-Bargmann space, Transactions AMS, 301 (1987), 813-829. MR0882716|(88c:47044)

$\left[\mathrm{BC}_{2}\right]$ C. A. Berger and L. A. Coburn, Heat flow and Berezin-Toeplitz estimates, American Journal of Mathematics, 116 (1994), 563-590. MR1277446 (95g:47038)

[E] M. Engliš, Compact Toeplitz operators via the Berezin transform on bounded symmetric domains. Integral Equations Operator Theory, 33 (1999), 426-455. MR1682815 (2000h:47050a)

[F] G. B. Folland, Harmonic analysis in phase space, Annals of Mathematics Studies, Princeton Univ. Press, Princeton, 1989. MR0983366 (92k:22017)

[G] V. Guillemin, Toeplitz operators in $n$ dimensions, Integral Equations and Operator Theory, 7 (1984), 145-205. MR0750217|(86i:58130)

[GK] I. C. Gohberg and M. G. Krein, Introduction to the theory of linear nonselfadjoint operators, Translations of Mathematical Monographs 18, American Mathematical Society, Providence, 1969. MR0246142 (39:7447)

[H] S. Helgason, Differential geometry, Lie groups, and symmetric spaces, Academic Press, New York, 1978. MR0514561 (80k:53081)

$[\mathrm{K}] \quad$ S. Krantz, Function theory of several complex variables, Wiley-Interscience, New York, 1982. MR0635928 (84c:32001)

[MPS] T. Mazur, P. Pflug and M. Skwarczynski, Invariant distances related to the Bergman function, Proceedings of the American Mathematical Society, 94 (1985), 72-76. MR781059 (86i:32047)

Department of Mathematics, SUny at Buffalo, Buffalo, New York 14260

E-mail address: lcoburn@acsu.buffalo.edu 